Trabajos Originales

\title{
HISTERECTOMÍA VAG INAL, ABDOMINAL Y VAGINAL ASISTIDA POR LAPAROSCOPIA: UN ANÁLISIS PROSPECTIVO Y ALEATORIO*
}

\author{
Gonzalo Vesperinas A. ${ }^{1}$, Carlos Rondini S. ${ }^{1}$, Carlos Troncoso $R .{ }^{1}$, Boris Morán $V .{ }^{1}$, Marco \\ Levancini A. ${ }^{1}$, César Avilés Z. ${ }^{1}$, Fernando Troncoso R. ${ }^{1}$ \\ ${ }^{1}$ Departamento de Ginecología, Hospital Padre Hurtado.
}

\section{RESUMEN}

Objetivo: Evaluar el resultado a corto plazo de tres técnicas de histerectomía. Pacientes: Se analizaron prospectivamente 117 pacientes sin prolapso uterino, ingresadas al Hospital Padre Hurtado entre Octubre 2000 y Julio 2003, las que se distribuyeron al azar y fueron sometidas a una de las tres técnicas de histerectomía: vaginal (HV), abdominal (HAT) y vaginal asistida por laparoscopia (HVLP). Resultados: El tiempo operatorio fue significativamente mayor en el grupo HVLA comparado con el de HAT $(95,5 \mathrm{v} / \mathrm{s} 74,0$ minutos, $p<0,001)$ y el de éste, a su vez, mayor que el del grupo de HV $(74,0 \mathrm{v} / \mathrm{s} 52,9$ minutos, $p<0,001)$. No hubo diferencias significativas en las complicaciones intraoperatorias ni en el requerimiento de drogas analgésicas. Las pacientes sometidas a HAT presentaron más complicaciones postoperatorias. La estada hospitalaria fue más prolongada en el grupo HAT comparado con los otros, entre los que no hubo diferencias. El costo de la HVLA fue mayor que el de la HAT y el de ésta superior al de la HV. Conclusión: Nuestros resultados señalan que la vía vaginal debe ser la primera elección en pacientes sin prolapso en casos seleccionados. La HAT debe considerarse para aquellas pacientes en que no esté indicado el abordaje vaginal o laparoscópico.

\section{PALABRAS CLAVES: Complicaciones quirúrgicas, histerectomía vaginal, histerectomía abdominal, histerectomía vaginal asistida por laparoscopía}

\section{SUMMARY}

We present a prospective analisis of three techniques of total hysterectomy in patients without uterine prolapse: vaginal (HV), abdominal (HAT) and laparoscopic assisted vaginal hysterectomy (HVLA). Objective: To evaluate short term outcome of three techniques of hysterectomy. Patients: 117 women admitted at Padre Hurtado Hospital between October 2000 and July 2003 were evaluated and randomly distributed to one of the three techniques above mentioned. Results: Surgical time was higher in the HVLA group when compared with the HAT $(95,5 \mathrm{v} / \mathrm{s} 74,0$ minutes, $p<0,001)$, and this one was higher than surgical time of the $\mathrm{HV}(74,0 \mathrm{v} / \mathrm{s} 52,9$ minutes, $\mathrm{p}<0,001)$. There were not differences in intraoperative complications nor in the need of analgesic drugs. The HAT group presented more postoperative complications. Hospital stay was longer in the HAT group than in the HVLA and HV, with no differences between them. HVLA cost was higher in HVLA than HAT and HV respectively. Conclusions: According to our results, vaginal approach

*Trabajo de ingreso del Dr. GonZALo VeSPERINAS, leído en la sesión del martes 5 de abril de 2005 de la Sociedad Chilena de Obstetricia y Ginecología. 
should be the first option for hysterectomy in patients without uterine prolapse in selected cases. HAT must be considered when vaginal or laparoscopic techniques are not indicated.

\section{KEY WORDS: Surgical complications, vaginal hysterectomy, abdominal hysterectomy, laparoscopic assisted vaginal hysterectomy}

\section{INTRODUCCIÓN}

La histerectomía es el procedimiento quirúrgico mayor más frecuente en la práctica ginecológica (1). Con el advenimiento de la técnica laparoscópica descrita por Reich en 1989 se reanudó la discusión acerca de la mejor vía de abordaje, desafiando las preferencias tradicionales: vaginal y abdominal $(2,3)$. Aún cuando la histerectomía laparoscópica se asocia a una recuperación más rápida que la abdominal, su mayor costo cuestiona su indicación. La histerectomía vaginal aparece entonces, como una alternativa válida (4).

El objetivo principal de este trabajo es evaluar los resultados a corto plazo y los costos directos de tres diferentes vías de abordaje quirúrgico de la histerectomía total en ausencia de prolapso uterino.

\section{MATERIAL Y MÉTODO}

Se analizaron prospectivamente 117 pacientes sin prolapso uterino, ingresadas al Hospital Padre Hurtado entre Octubre 2000 y Julio 2003, las que se distribuyeron al azar y fueron sometidas a una de las tres técnicas de histerectomía: vaginal $(H V=38)$, abdominal $(H A T=43)$ y vaginal asistida por laparoscopía (HVLP=36).

Selección de las pacientes. Las pacientes fueron ingresadas desde el Centro de Referencia de Salud del Hospital Padre Hurtado. Todas aquellas que cumplieron con los criterios de inclusión (Tabla I) y que firmaron el consentimiento informado fueron incluidas en el estudio. Un total de 123 pacientes fue seleccionadas de acuerdo a los criterios de inclusión. De ellas, 6 fueron eliminadas del análisis: 2 no firmaron el consentimiento informado; 1 desistió de la cirugía; en 2 casos se extravió la información y 1 paciente sometida a histerectomía vaginal asistida por laparoscopía se convirtió a técnica abdominal (la conversión se debió al hallazgo de adherencias muy importantes al momento de la introducción de la óptica, por lo que se decidió cambiar la vía de inmediato).

Distribución aleatoria. La fecha de cirugía y el equipo médico fueron determinados al momento del ingreso. Un listado aleatorio de las tres técnicas fue obtenido computacionalmente (programa Epi-Info 2000, Atlanta, Georgia, USA), asignándose posteriormente a cada una de las pacientes una de las alternativas quirúrgicas. Todas las intervenciones fueron realizadas por el equipo de ginecología del Hospital Padre Hurtado.

Técnica quirúrgica. En todas las HVLA se usó anestesia general. En los abordajes vaginal y abdominal se usó anestesia regional. La técnica operatoria en la HAT fue la descrita por Richardson (5). Para la HV se utilizó la técnica de Nichols (6). En el caso de la HVLA se realizaron incisiones de $10 \mathrm{~mm}$ (umbilical) y tres accesorias de $5 \mathrm{~mm}$ en hipogastrio y fosas ilíacas. Se mantuvo una presión de $\mathrm{CO}_{2}$ de $15 \mathrm{mmHg}$ y se realizó la liberación

\section{Tabla I}

\section{CRITERIOS DE INCLUSIÓN Y EXCLUSIÓN}

\footnotetext{
A. Criterios de inclusión

Paciente con indicación de histerectomía:

- Leiomiomas uterinos.

- Patología endometrial

- Sospecha de adenomiosis sintomática.

- Dolor pélvico crónico de origen uterino.

- LIE de alto grado persistente.

- Consentimiento informado firmado

B. Criterios de exclusión

Sospecha de adherencias peritoneales firmes:

- Antecedente de peritonitis de cualquier origen.

- Antecedente de absceso tuboovárico.

- Antecedente o sospecha de endometriosis.

- Indicación de cirugía sobre los anexos:

- Presencia de tumor anexial.

- Presencia de prolapso uterino grado II o III.

- Contraindicaciones generales de la técnica laparoscópica:

- Enfermedad respiratoria grave.

- Dos o más cirugías abdominales previas.

- Contraindicaciones generales de la vía vaginal:

- Pacientes sin actividad sexual.

- Radioterapia local.

- Vagina infundibuliforme.

- Diámetro transverso uterino mayor de $10 \mathrm{~cm}$ a la ecografía.

- Pacientes que no hayan firmado el consentimiento informado.
} 
de los anexos y la disección de los ligamentos anchos y del espacio vésico-uterino con coagulación bipolar y tijeras. En el tiempo vaginal se completó la cirugía, incluyendo la ligadura de los pedículos vasculares uterinos.

Principales variables a medir. Como variables principales se analizó tasa y tipo de complicaciones intra y postoperatorias. La hemorragia intraoperatoria se definió como aquella pérdida hemática que requiriera transfusión o la que ocasionara un descenso del hematocrito de 10 o más puntos. Además, se compararon los tiempos operatorios, los días de persistencia del dolor postoperatorio (referidos por las pacientes en los controles ambulatorios), requerimiento de drogas analgésicas (fármacos y dosis), la estada hospitalaria (en horas, desde el inicio de la intervención hasta el alta del hospital) y costo total del tratamiento. Tanto la indicación de drogas analgésicas como el alta hospitalaria fueron determinadas de acuerdo a criterios preestablecidos (Tablas II y III). Para la evaluación del dolor se utilizó la escala visual análoga (EVA), anotando los resultados cada hora durante las primeras seis y luego a intervalos de tres horas durante el primer día y de seis durante los días siguientes, hasta el alta. Para el cálculo del valor de las prestaciones se incluyeron los costos directos (recursos humanos, insumos, medicamentos, dependientes de equipos, alimentación de pacientes, esterilización) y los indirectos, como consumos básicos y convenios externos, entre otros. También se agregó el costo de administración y gerencia.

Se dispuso de un formato de registro especialmente diseñado para la investigación, que se adjuntó a la ficha clínica de la paciente.

Análisis estadístico. Para realizar comparaciones de variables categóricas se utilizó el test de chi cuadrado. Cuando se compararon variables categóricas con variables continuas se utilizó el test de ANOVA. El test de comparaciones múltiples para estas últimas fue el de Bonferroni. Para el análisis de los datos se usó el programa estadístico SPSS ${ }^{\circledR}$ (SPSS 12.0 para Windows, The Apache Software Foundation, 2000). Para todas

\section{Tabla III}

\section{CRITERIOS DE ALTA HOSPITALARIA}

- Buen estado general de la paciente.

- Escaso dolor abdominal espontáneo (EVA menor de 5).

- Buena tolerancia a la alimentación oral con régimen liviano.

\section{Tabla II}

\section{CRITERIOS DE INDICACIÓN DE DROGAS ANALGÉSICAS}

- Durante las primeras 24 horas se administrará Morfina $3 \mathrm{mg}$ IM si EVA > 5 .

- Durante los días restantes se administrará Diclofenaco $50 \mathrm{mg}$ oral si EVA > 5. De presentarse dolor importante, se administrará Metadona $0,1 \mathrm{mg} / \mathrm{K}$ de peso IV, de acuerdo a los requerimientos.

las variables fue considerado como significativo un $\mathrm{p}<0,05$.

\section{RESULTADOS}

Las características de la muestra se detallan en la Tabla IV. No hubo diferencias significativas entre los grupos. Nuestro universo correspondió, en general, a pacientes multíparas, premenopáusicas y con un sobrepeso importante.

Un $75 \%$ de nuestras pacientes se intervino por leiomiomas sintomáticos; un $11 \%$ por patología endometrial (hiperplasia, pólipos, etc.) y $8 \%$ por algia pélvica como indicación principal (Tabla V).

El resultado operatorio y la estada hospitalaria se resumen en la Tabla VI. No hubo diferencias en el peso uterino ni en el descenso del hematocrito. La HVLA se asoció a un tiempo operatorio significativamente mayor que la HAT $(95,5 \mathrm{v} / \mathrm{s} 74,0 \mathrm{mi}-$ nutos, $p<0,001)$ y ésta, a su vez, fue más lenta que la HV $(74,0 \mathrm{v} / \mathrm{s} 52,9$ minutos, $\mathrm{p}<0,001)$. La estada hospitalaria fue significativamente mayor en el caso de la HAT comparada con HVLA y HV, no encontrándose diferencias entre estas últimas.

En cuanto al dolor postoperatorio, hubo una tendencia a mayor requerimiento de drogas analgésicas (morfina y AINE) en las pacientes sometidas a HAT, pero sin alcanzar significancia estadística. En concordancia con lo anterior, el puntaje de EVA fue mayor (aunque no significativo) en las pacientes operadas por vía abdominal. En las primeras horas de postoperatorio el dolor fue mayor en las pacientes operadas vía laparoscópica (Figura 1). Posteriormente fue el grupo de HAT el que tuvo tendencia a presentar el más alto puntaje de EVA. Se observó un aumento del dolor durante el segundo día en todos los grupos (Figura 2).

Las complicaciones intraoperatorias fueron hemorragia ( 6 casos) y daño vesical ( 2 casos). Estos últimos consistieron en perforación vesical y ambos ocurrieron en el grupo de HVLA, al disecar el 
Tabla IV

CARACTERÍSTICAS DE LA POBLACIÓN

\begin{tabular}{|c|c|c|c|c|c|c|c|}
\hline & \multicolumn{2}{|c|}{$\operatorname{HVLA}(n=36)$} & \multicolumn{2}{|c|}{$H V(n=38)$} & \multicolumn{2}{|c|}{ HAT $(n=43)$} & \multirow[t]{2}{*}{$p$} \\
\hline & $x$ & $(D S)$ & $x$ & $(D S)$ & $x$ & $(D S)$ & \\
\hline Edad (años) & 44,7 & $(4,5)$ & 44,7 & $(6,3)$ & 44,9 & $(6,2)$ & n.s. \\
\hline Peso (kg) & 69,1 & $(12,1)$ & 71,7 & $(10,5)$ & 68,1 & $(10,5)$ & n.s. \\
\hline Talla (cm) & 154,7 & $(5,8)$ & 155,8 & $(5,5)$ & 154,1 & $(5,8)$ & n.s. \\
\hline IMC & 28,9 & $(5,0)$ & 29,5 & $(3,9)$ & 28,7 & $(4,3)$ & n.s. \\
\hline Partos (n) & 2,9 & $(1,0)$ & 3,6 & $(1,4)$ & 3,0 & $(1,8)$ & n.s. \\
\hline Cirugías (n) & 0,4 & $(0,5)$ & 0,6 & $(0,7)$ & 0,5 & $(0,5)$ & n.s. \\
\hline Hematocrito (\%) & 35,4 & $(4,8)$ & 35,4 & $(4,6)$ & 36,6 & $(4,3)$ & n.s. \\
\hline
\end{tabular}

$\mathrm{X}=$ promedio.

$\mathrm{DS}=$ desviación estándar.

$\mathrm{ns}=$ no significativo

Tabla V

INDICACIÓN OPERATORIA

\begin{tabular}{lrr}
\hline & $n$ & $\%$ \\
\hline Leiomioma & 88 & 75 \\
Patología endometrial & 13 & 11 \\
Algia pélvica & 9 & 8 \\
Metrorragia & 3 & 3 \\
Adenomiosis & 3 & 3 \\
Patología cervical & 1 & 1 \\
\hline
\end{tabular}

espacio vesicouterino en el tiempo laparoscópico. En las dos ocasiones se reparó la vejiga en el tiempo vaginal. Si bien existió una tendencia a tener menos complicaciones en la HAT, ésta no fue significativa (Tabla VII). Ninguna de las compli-

\section{Dolor Postoperatorio}

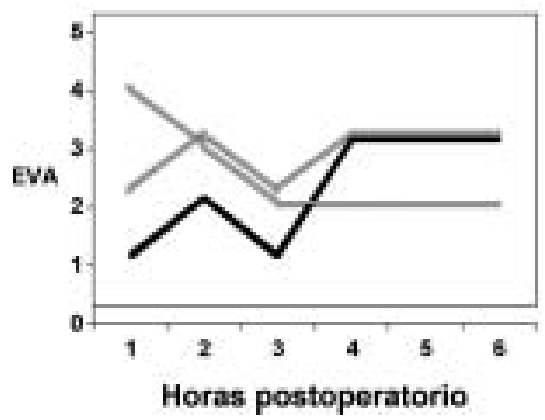

HVLA: Histerectomía vaginal laparoscópicamente asistida. HV: Histerectomía vaginal.

HAT: Histerectomía abdominal total.

Figura 1. Dolor postoperatorio inmediato según escala visual análoga (EVA).
Tabla VI

RESULTADOS POST OPERATORIOS

\begin{tabular}{lrrrr}
\hline & HVLA & HV & HAT & \\
& $(n=36)$ & $(n=38)$ & $(=43)$ & $p$ \\
\hline Peso uterino (g) & 243,2 & 210,3 & 243,0 & n.s. \\
Tiempo operatorio (min) & 95,8 & 52,9 & 74,0 & n.s. \\
Caída de hematocrito (\%) & 4,2 & 4,3 & 2,8 & n.s. \\
Estada hospitalaria (horas) & 54,6 & 57,6 & 71,4 & n.s. \\
\hline n.s. = no significativo. & & & &
\end{tabular}

caciones descritas requirió de conversión de la vía de abordaje para su resolución.

Las complicaciones postoperatorias se detaIlan en la Tabla VIII. Todas fueron menores y afectaron a 9 pacientes sometidas a HAT, a 5 opera-

\section{Dolor Postoperatorio}

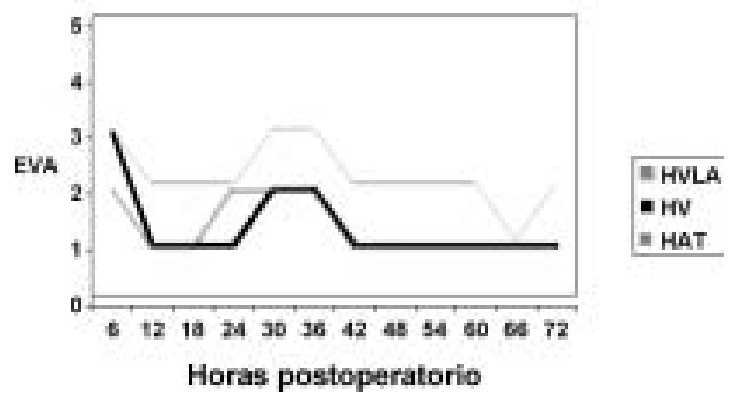

HVLA: Histerectomía vaginal laparoscópicamente asistida. HV: Histerectomía vaginal.

HAT: Histerectomía abdominal total.

Figura 2. Dolor postoperatorio tardío según escala visual análoga (EVA). 
Tabla VII COMPLICACIONES INTRAOPERATORIAS

\begin{tabular}{lcccc}
\hline & $\begin{array}{c}\text { HVLA } \\
(n=36)\end{array}$ & $\begin{array}{c}\text { HV } \\
(n=38)\end{array}$ & $\begin{array}{c}\text { HAT } \\
(n=43)\end{array}$ & $p$ \\
\hline Hemorragia $(\mathrm{n})$ & 2 & 3 & 1 & \\
Perforación vesical $(\mathrm{n})$ & 2 & 0 & 0 & \\
\hline Total & 4 & 3 & 1 & n.s.
\end{tabular}

$\mathrm{n}=$ número de pacientes.

n.s.= no significativo.

das de HVLA y sólo a una luego de HV. En ninguno de los casos fue necesario hospitalizar a la paciente, logrando la resolución del problema con manejo ambulatorio en un máximo de dos semanas.

El valor total actualizado al primer semestre de 2004 para HVLA fue de $\$ 423.987$, HAT de $\$$ 312.179 y HV de $\$ 277.493$.

\section{DISCUSIÓN}

La histerectomía es un procedimiento quirúrgico frecuente. Anualmente se operan 600.000 mujeres en Estados Unidos, siendo ésta la segunda cirugía en frecuencia en mujeres en edad reproductiva (1), sólo superada por la cesárea.

Se discute si la vía de abordaje obedece a razones técnicas o se debe a preferencias del equipo médico (7). Tradicionalmente la más usada ha sido la vía abdominal, que confiere un campo quirúrgico más amplio que la vaginal. Esta ventaja se ve contrarrestada por un mayor dolor postoperatorio y una estada hospitalaria más larga, aún cuando se trate de pacientes con útero voluminoso (8). Con el advenimiento de la técnica laparoscópica se pretende aprovechar las ventajas de la visión que se obtiene por vía abierta con el mínimo trauma quirúrgico asociado a la vía vaginal. Yuen y colaboradores han comunicado que en la histerectomía laparoscópica se ocasiona un menor daño tisular y una menor respuesta de estrés que la histerectomía abdominal, aunque esto no ha sido corroborado por otros $(9,10)$. Por otra parte, la técnica laparoscópica tiene un costo mayor que la abdominal y que la vaginal. Si bien éste puede reducirse al no utilizar material desechable, el costo del equipo y el mayor tiempo operatorio hacen que se mantenga un valor total más elevado, de acuerdo a cifras norteamericanas $(4,11)$. Nuestros resultados demuestran que la ci-
Tabla VIII COMPLICACIONES POSTOPERATORIAS

\begin{tabular}{lccc}
\hline & $\begin{array}{c}\text { HVLA } \\
(n=36)\end{array}$ & $\begin{array}{c}H V \\
(n=38)\end{array}$ & $\begin{array}{c}H A T \\
(n=43)\end{array}$ \\
\hline Flujo vaginal patológico $(\mathrm{n})$ & 2 & 0 & 2 \\
Granuloma de cúpula $(\mathrm{n})$ & 0 & 1 & 2 \\
Infección de herida $(\mathrm{n})$ & 1 & 0 & 1 \\
Hematoma de herida $(\mathrm{n})$ & 1 & 0 & 1 \\
Seroma de herida $(\mathrm{n})$ & 0 & 0 & 1 \\
Fiebre (n) & 0 & 0 & 1 \\
Algia pélvica $(\mathrm{n})$ & 0 & 0 & 1 \\
Infección urinaria $(\mathrm{n})$ & 1 & 0 & 0 \\
\hline Total & 5 & $1^{*}$ & $9^{*}$
\end{tabular}

$\mathrm{n}=$ número de pacientes; ${ }^{*} \mathrm{p}<0,001$.

rugía laparoscópica tiene un costo mayor que el de las dos alternativas. La histerectomía vaginal resultó tener el menor valor, considerando una estada hospitalaria más breve si se la compara con las pacientes sometidas a HAT.

Nuestra tasa de complicaciones intra y postoperatorias no difiere de otras series (12). Los tiempos operatorios y pesos uterinos están en el rango de lo publicado por otros grupos $(13,14)$. El mayor dolor en las primeras horas de postoperatorio en las pacientes operadas vía laparoscópica se explica por el uso de anestesia general en este grupo, con un efecto residual postoperatorio menor en comparación con las pacientes sometidas a HAT y a HV, en las que se utilizó anestesia regional. El aumento del dolor observado durante el segundo día en todos los grupos puede obedecer al cambio en el tipo de fármacos, pasando desde los opiáceos a los AINE. Nuestra conducta posterior ha sido el uso horario de AINE durante el segundo día postoperatorio, para evitar este efecto.

\section{CONCLUSIONES}

Nuestros resultados indican que la vía vaginal presenta ventajas al compararla con la abdominal y la laparoscópica. En nuestro centro la histerectomía vaginal es la primera elección, recurriendo a la asistencia laparoscópica cuando se requiere exploración anexial y/o anexectomía. La vía abdominal se reserva para aquellos casos en que no es recomendable el uso de las técnicas anteriores. Queda por definir si se amplían o no los criterios de selección de las pacientes para la vía vaginal, especialmente el del número de cirugías abdominales previas. 


\section{BIBLIOGRAFÍA}

1. Lepine LA, Hillis SD, Marchbanks PA et al. Hysterectomy surveillance-United States, 1980-1993. In: CDC surveillance summaries (August 8 ). MMWR 1997; 46(№ SS-4); 1-16.

2. Kovac SR. Guidelines to determine the route of hysterectomy. Obstet Gynecol 1995; 85(1): 18-23.

3. Harkki-Siren P, Sjoberg J, Makinen J et al. Finnish national register of laparoscopic hysterectomies: a review and complications of 1165 operations. Am J Obstet Gynecol 1997; 176(1): 118-22.

4. Dorsey JH, Holtz PM, Griffiths RI, McGrath MM, Steinberg EP. Costs and charges associated with three alternative techniques of hysterectomy. $\mathrm{N}$ Engl J Med 1996; 335(7): 476-82.

5. Thompson JD, Rock JA. Te Linde's Operative Gynecology, 7th ed. Lippincott Co. 1992.

6. Nichols DH, Randall CL. Vaginal surgery, $3^{\text {rd }}$ ed. Williams \& Wilkins,1989; Cap. 10: 182-238.

7. Dorsey JH, Steinberg EP. Clinical indications for hysterectomy route: patient characteristics or physician preference? Am J Obstet Gynecol 1995; 173(5): 1452-60.

8. Benassi L, Rossi T, Kaihura CT et al. Abdominal or vaginal hysterectomy for enlarged uteri: a randomized clinical trial. Am J Obstet Gynecol 2002; 187: 1561-5.
9. Yuen PM, Mack TW, Yim SF et al. Metabolic and inflammatory responses after laparoscopic and abdominal hysterectomy. Am J Obstet Gynecol 1998; 179: 1-5.

10. Ellström $M$, Bengtsson A, Tylman $M$, Haeger $M$, Olsson $\mathrm{JH}$, Hahlin M. Evaluation of tissue trauma after laparoscopic and abdominal hysterectomy: measurements of neutrophil activation and release of interleukin-6, cortisol and c-reactive protein. J Am Coll Surg 1996; 182: 423-30.

11. Summitt R, Stovall T, Lipscomb GH, Ling FW. Randomized comparison of laparoscopic assisted vaginal hysterectomy with standard vaginal hysterectomy in an outpatient setting. Obstet Gynecol 1992; 80: 895-901.

12. Mäkinen J, Johansson J, Tomás C et al. Morbidity of 10110 hysterectomies by tipe of approach. Human Reprod 2001; 16(7): 1473-8.

13. Ottosen C, Lingman G, Ottosen L. Three methods for hysterectomy: a randomized, prospective study of short term outcome. BJOG 2000; 107(11): 13805.

14. Ribeiro SC, Ribeiro RM, Santos NC, Pinotti JA. A randomized study of total abdominal, vaginal and laparoscopic hysterectomy. Int J Obstet Gynecol 2003; 83: 37-43. 